\title{
Computational evaluation of the flexoelectric effect in dielectric solids
}

\author{
Amir Abdollahi, Christian Peco, Daniel Millán, Marino Arroyo, and Irene Arias ${ }^{a}$ ) \\ Laboratori de Càlcul Numèric (LaCàN), Universitat Politècnica \\ de Catalunya (UPC), Campus Nord UPC-C2, E-08034 Barcelona, \\ Spain.
}

(Dated: 6 October 2014) 
Flexoelectricity is a size-dependent electromechanical mechanism coupling polarization and strain gradient. It exists in a wide variety of materials, and is most noticeable for nanoscale objects, where strain gradients are higher. Simulations are important to understand flexoelectricity because experiments at very small scales are difficult, and analytical solutions are scarce. Here, we computationally evaluate the role of flexoelectricity in the electromechanical response of linear dielectric solids in two-dimensions. We deal with the higher-order coupled partial differential equations using smooth meshfree basis functions in a Galerkin method, which allows us to consider general geometries and boundary conditions. We focus on the most common setups to quantify the flexoelectric response, namely bending of cantilever beams and compression of truncated pyramids, which are generally interpreted through approximate solutions. While these approximations capture the size-dependent flexoelectric electromechanical coupling, we show that they only provide order-of-magnitude es-
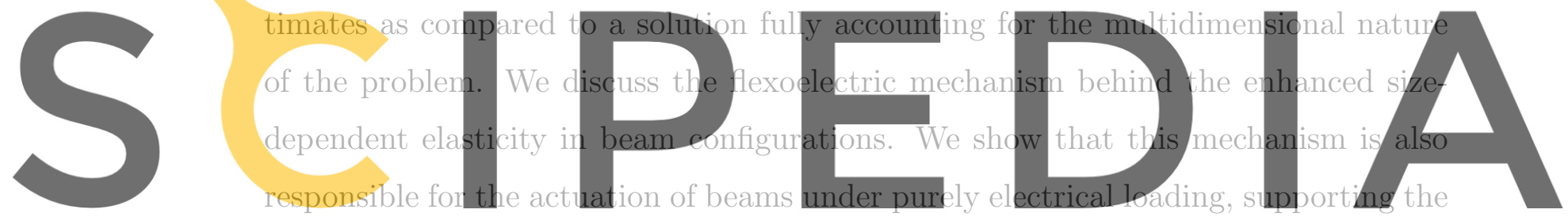
Register for free at httpș//wwW.scipedia.com to download the version without the watermark actuation-induced curvature is in a good agreement with experimental results. The

truncated pyramid configuration highlights the critical role of geometry and boundary conditions on the effective electromechanical response. Our results suggest that computer simulations can help understanding and quantifying the physical properties of flexoelectric devices.

\footnotetext{
a)http://www.lacan.upc.edu; Electronic mail: irene.arias@upc.edu
} 


\section{INTRODUCTION}

Since its introduction by Mashkevich and Tolpygo ${ }^{1}$, flexoelectricity has been identified as an important electromechanical coupling in a wide variety of materials, including cellular membranes, liquid crystals, polymers, graphene, and piezoelectric and non-piezoelectric crystals $^{2}$. With the emergence of nanoscale fabrication and characterization, the interest in the flexoelectric effect has acquired a renewed vitality. See Refs. ${ }^{2-4}$ for recent reviews. Phenomenologically, the flexoelectric effect describes the generation of an electric polarization induced by strain gradient:

$$
P_{i}=\mu_{i j k l} \nabla_{l} \varepsilon_{j k}
$$

where $\mathbf{P}$ is the electric polarization, $\varepsilon$ is the mechanical strain, and $\mu$ is a fourth order flexoelectric tensor. Two features make flexoelectricity distinct from other electromechanical coupling mechanisms such as piezoelectricity. The first feature is its universality, due to the

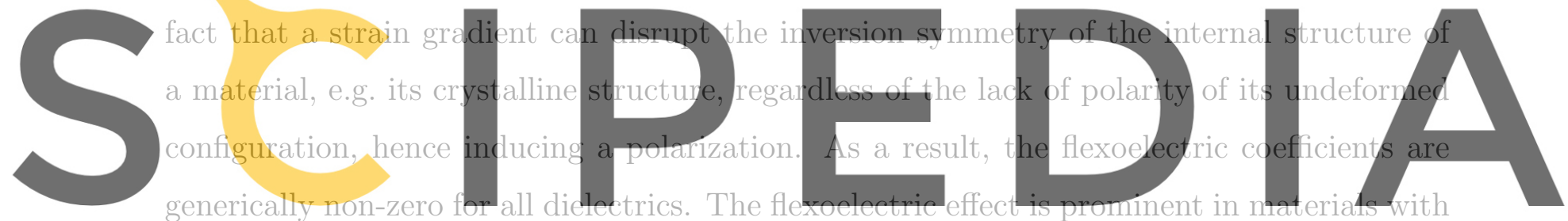

Register fop free at https/NwWw.scipedia.com to download the version without the watermark it can only appear in non-centrosymmetric crystals. The second distinguishing feature of

flexoelectricity is its size-dependence, due to the scaling of strain gradients with structural size. Despite its universality, the flexoelectric effect is typically insignificant relative to piezoelectricity at macroscopic scales, and only manifests itself noticeably at the nanoscale. For this reason, the experimental observation of flexoelectricity is particularly difficult, which motivates the development of theoretical models to investigate this phenomenon.

A number of theoretical studies have focused on understanding the flexoelectric behavior of dielectrics. Kogan presented the first phenomenological theory and provided a rough theoretical estimate of the flexoelectric coefficient ${ }^{11}$. The first comprehensive study is by Tagantsev $^{12}$, who clarified the distinction between piezoelectric and flexoelectric responses. Refinements of this theory have including connections with core-shell lattice models ${ }^{4,13}$ and the contribution of surface piezoelectricity ${ }^{14}$. A mathematical framework for the governing equations of flexoelectricity and some simple solutions was proposed subsequently ${ }^{15}$. Following this work, Sharma and coworkers ${ }^{16,17}$ presented a theoretical analysis on the role 
of flexoelectricity in both piezoelectric and non-piezoelectric nanostructures, particularly in nanoscale cantilever beams. They also carried out atomistic calculations to verify the analytical results, showing a considerable enhancement and size-dependence of the apparent piezoelectricity and elasticity in nanostructures. Based on this theoretical framework, it has also been demonstrated that (1) nanocomposites and superlattices can be effectively piezoelectric without using piezoelectric materials ${ }^{18,19}$ and (2) a dramatic enhancement can be achieved in energy harvesting in piezoelectric nanostructures ${ }^{20}$. Cross and coworkers presented a simple formulation to estimate the effective piezoelectric constant of a dielectric truncated pyramid under compression accounting for flexoelectricity ${ }^{21,22}$. The influence of the flexoelectric effect on the electromechanical properties of bending nanobeams has been recently investigated ${ }^{23-25}$. Within the Landau-Ginsburg-Devonshire phenomenological approach, it has been shown that the flexoelectric coupling influences the majority of properties of nanoferroics ${ }^{26}$. Catalan and coworkers presented a phenomenological model of the effect

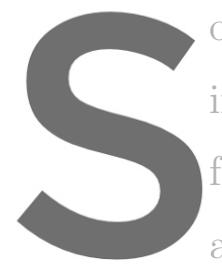
of flexoelectricity on the properties of ferroelectric thin films?. The surface effects have beep
introduced in a theoretical model of fexpelectricity for dielectrics, accounting for both sur
face stress and surface polarigation 27 Nlexoelectric propertids of crystalline dielectrics have
also been estimated using atonistic calculations

to connect density-functional calculations with continuum flexoelectricity ${ }^{32,33}$. However, a Register for free at https//www.scipedia.com to download the version without the watermark direct comparison of first-principles calculations with experimentally measured coefficients

is still $\operatorname{far}^{3}$, due for instance to finite temperature effects or to finite sample effects such as surface piezoelectricity, difficult to untangle from bulk contributions ${ }^{14,34}$.

Focusing on the above-mentioned phenomenological continuum theory, the resulting fourth-order coupled system of partial differential equations (PDEs) has been approached with analytical solutions relying on simplifying assumptions and in very simple geometries (cantilever beams under bending ${ }^{16,17,23}$, truncated pyramids under compression ${ }^{21,22}$ ). In addition, to interpret experiments, the two-way flexoelectric coupling is often ignored, by estimating strain gradients from elasticity alone $\mathrm{e}^{8}$. The approximate solutions under restrictive assumptions may lead to under- or over-estimation of the flexoelectric effect. Moreover, flexoelectricity can be more prominent in complex geometries favoring strain gradients, for which analytical solutions are not available (see Ref. ${ }^{35}$ for recent efforts to provide analytical solutions for some flexoelectric boundary value problems). More recently, within the Ginzburg-Landau framework, a number of numerical phase-field simulations have been 
performed to evaluate the effect of flexoelectricity on domain patterns and domain walls in ferroelectrics ${ }^{36-38}$. However, these references resort to finite difference methods with uniform grids, which are limited to very simple geometries and boundary conditions. We are not aware of previous numerical approaches to solve the boundary value problems of flexoelectricity, treating the multidimensionality of the coupled fields in general geometries with different boundary conditions. The main difficulty is the fourth order nature of the PDEs of flexoelectricity, which demands at least $C^{1}$ continuous basis functions for a direct Galerkin method. Alternatively, mixed finite elements only requiring $C^{0}$ continuity and previously developed for strain gradient elasticity ${ }^{39}-11$ could be applied to flexoelectricity. Here, we resort to local maximum-entropy (LME) meshfree approximants ${ }^{42}$. The basis functions exhibit $C^{\infty}$ smoothness, and therefore a straight Galerkin approach is possible. LME approximants have been successfully applied to a variety of problems with high-order functionals including biomembrane phase-field models ${ }^{43,44}$, or thin-shells $s^{45,46}$.

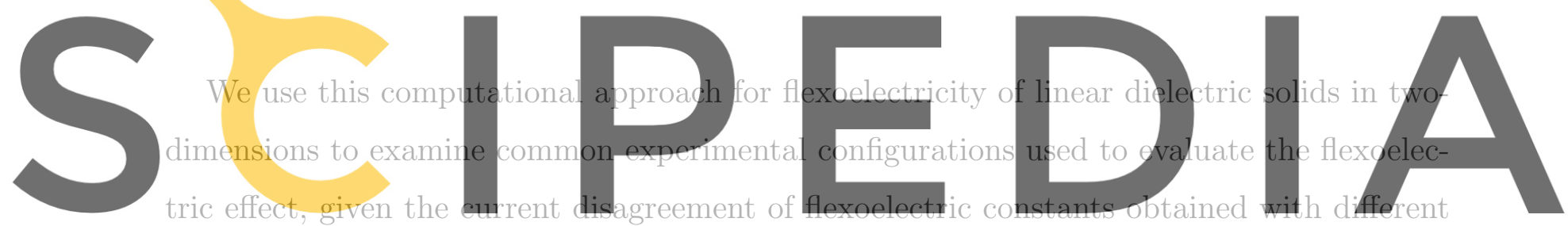

Register for free athiths W//wWW.scipedia.com to download the version without the watermark current simple estimations may be one of the sources of current controversies in material

characterization $^{3}$. Another controversy in flexoelectricity is even more profound, as its existence as a bulk effect has been put into question, favoring an explanation of experiments through surface effects ${ }^{14,34}$. A proper investigation of this issue demands an accurate solution of the bulk equations. We do not address this controversy here, but our simulation methodology provides tools for this debate.

A continuum theory of flexoelectricity is presented in Section II. We then perform simulations of a cantilever beam under bending and of a truncated pyramid under compression, which are the most common configurations to assess the flexoelectric response of dielectric solids. The results for the beam with different boundary conditions are reported in Section III A along with a comparison with analytical and experimental results. Section III B presents the results for truncated pyramids, which are compared with previous estimations. Section IV summarizes the main results of the paper. 


\section{THEORY}

We summarize next a linear theory of flexoelectricity previously proposed in Refs. ${ }^{17,19}$ and references therein. The electrical enthalpy density of a linear dielectric solid possessing piezoelectricity and flexoelectricity can be written as

$$
\begin{aligned}
\mathcal{H}\left(\varepsilon_{i j}, E_{i}, \varepsilon_{j k, l}, E_{i, j}\right) & =\frac{1}{2} \mathbb{C}_{i j k l} \varepsilon_{i j} \varepsilon_{k l}-e_{i k l} E_{i} \varepsilon_{k l}+f_{i j k l} E_{i} \varepsilon_{j k, l} \\
& +d_{i j k l} E_{i, j} \varepsilon_{k l}-\frac{1}{2} \kappa_{i j} E_{i} E_{j},
\end{aligned}
$$

where $E_{i}=-\phi_{, i}$ is the electric field, $\phi$ being the electric potential. The first energy term is the elastic potential, where $\mathbb{C}$ is the fourth-order tensor of elastic moduli. The piezoelectric coupling between the strain and electric field is through the second term with the third-order tensor of piezoelectricity e. The last energy term is the electrostatic contribution, where $\kappa$ is the second-order dielectric tensor. Here, our particular attention is on the third and fourth terms, which define the flexoelectric behavior of the material. The term coupling

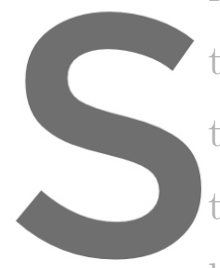
the gradient of strain $\nabla \varepsilon$ to the electric fic
the fourth-order tens or $\mathrm{f}$. Conversely, the
through the fourth-order tensor d, termed
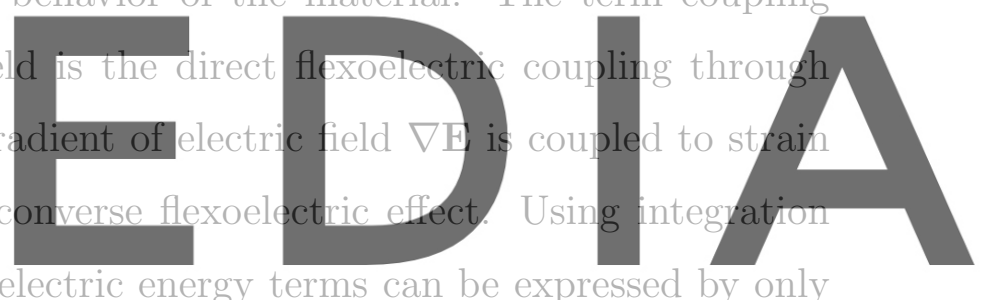

by parts, it has been shown that these flexoelectric energy terms can be expressed by only

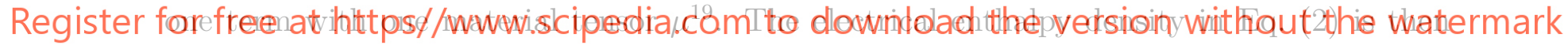

rewritten as

$$
\mathcal{H}\left(\varepsilon_{i j}, E_{i}, \varepsilon_{j k, l}\right)=\frac{1}{2} \mathbb{C}_{i j k l} \varepsilon_{i j} \varepsilon_{k l}-e_{i k l} E_{i} \varepsilon_{k l}-\mu_{i j k l} E_{i} \varepsilon_{j k, l}-\frac{1}{2} \kappa_{i j} E_{i} E_{j},
$$

where $\mu_{i j k l}=d_{i k l j}-f_{i j k l}$. See Refs. ${ }^{47,48}$ for recent accounts on the symmetry of the tensor of flexoelectric coefficients. The two forms of the enthalpy density in Eqs. (2) and (3) result in identical governing equations, and only the associated natural boundary conditions are different. We ignore strain gradient elasticity for simplicity and to isolate the effect of flexoelectricity, although as argued in Ref. ${ }^{35}$ and later in this paper, this may compromise the stability of the model in some regimes.

Defining the usual stress and electric displacements

$$
\hat{\sigma}_{i j}=\frac{\partial \mathcal{H}}{\partial \varepsilon_{i j}}, \quad \hat{D}_{i}=-\frac{\partial \mathcal{H}}{\partial E_{i}},
$$

and the higher-order (hyper) stress and electric displacements arising from flexoelectricity,

$$
\widetilde{\sigma}_{i j k}=\frac{\partial \mathcal{H}}{\partial \varepsilon_{i j, k}}, \quad \widetilde{D}_{i j}=-\frac{\partial \mathcal{H}}{\partial E_{i, j}},
$$


the physical stresses $\sigma$ and the physical electric displacements $\mathbf{D}$ emerging from the theory can be written as

$$
\sigma_{i j}=\hat{\sigma}_{i j}-\widetilde{\sigma}_{i j k, k}=\mathbb{C}_{i j k l} \varepsilon_{k l}-e_{k i j} E_{k}+\mu_{l i j k} E_{l, k}
$$

and

$$
D_{i}=\hat{D}_{i}-\widetilde{D}_{i j, j}=e_{i k l} \varepsilon_{k l}+\kappa_{i j} E_{j}+\mu_{i j k l} \varepsilon_{j k, l}
$$

In these equations we have assumed that the material properties are uniform. The last term in Eq. (6) is a mechanical stress induced by gradients of the electric field, while the last term in Eq. (7) is the induced polarization in Eq. (1) due to flexoclectricity.

The physical interpretation of the higher-order stresses is open, and as mentioned earlier it relates to the electromechanical boundary conditions ${ }^{26}$. In the model in Eq. (2), both higherorder stresses and electric displacements affect the natural boundary conditions, while the model in Eq. (3) does not have a higher-order electric displacement contribution. Therefore,
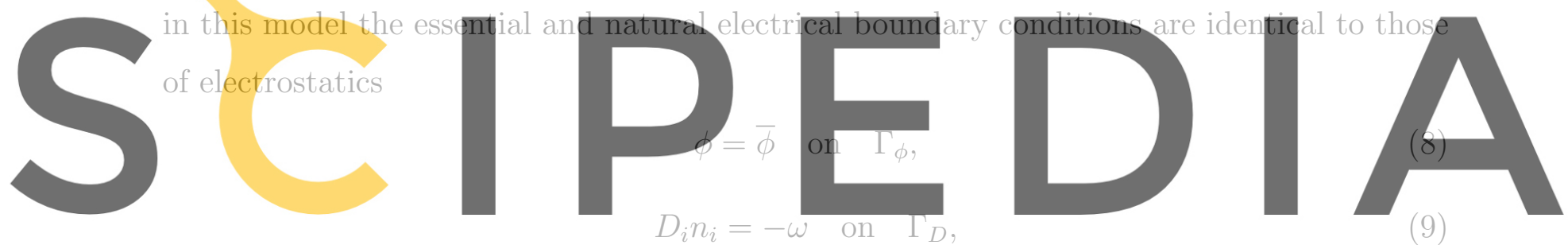

Register for free at https//www.scipedia.com to download the version without the watermark

$\partial \Omega$ is the boundary of the domain $\Omega$ with unit normal $n_{i}$.

As for the mechanical boundary conditions, either displacement or traction need to be specified:

$$
\begin{gathered}
u_{i}=\bar{u}_{i} \text { on } \Gamma_{u}, \\
t_{k}=n_{j}\left(\hat{\sigma}_{j k}-\widetilde{\sigma}_{i j k, i}\right)-D_{j}\left(n_{i} \widetilde{\sigma}_{i j k}\right)-\left(D_{p} n_{p}\right) n_{i} n_{j} \widetilde{\sigma}_{i j k}=\bar{t}_{k} \text { on } \Gamma_{t},
\end{gathered}
$$

where $\bar{u}_{i}$ and $\bar{t}_{k}$ are the prescribed mechanical displacements and tractions, $D_{j}=\partial_{j}-n_{j} D^{n}$ is the surface gradient operator, $D^{n}=n_{k} \partial_{k}$ is the normal gradient operator, $\Gamma_{u} \cup \Gamma_{t}=\partial \Omega$, and $\Gamma_{u} \cap \Gamma_{t}=\varnothing$. It is clear that the traction boundary condition in Eq. (11) is affected by the higher-order stresses. In addition to these, the strain gradients result in other types of boundary conditions as ${ }^{35}$ :

$$
\begin{gathered}
u_{i, j} n_{j}=\bar{v}_{i} \quad \text { on } \quad \Gamma_{v}, \\
n_{i} n_{j} \widetilde{\sigma}_{i j k}=\bar{r}_{k} \quad \text { on } \quad \Gamma_{r},
\end{gathered}
$$


where $\bar{v}$ is the prescribed normal derivative of displacement, $\bar{r}_{k}$ is the higher-order traction, $\Gamma_{v} \cup \Gamma_{r}=\partial \Omega$, and $\Gamma_{v} \cap \Gamma_{r}=\varnothing$. Here, we assume homogeneous natural boundary conditions on $\Gamma_{v}$ and $\Gamma_{r}$, i.e. $\bar{v}_{i}=\bar{r}_{k}=0$.

The total electrical enthalpy can then be written as

$$
H=\frac{1}{2} \int_{\Omega}\left(\hat{\sigma}_{i j} \varepsilon_{i j}+\widetilde{\sigma}_{i j k} \varepsilon_{i j, k}-\hat{D}_{i} E_{i}\right) \mathrm{d} \Omega-\int_{\Gamma_{t}} \bar{t}_{i} u_{i} \mathrm{~d} S+\int_{\Gamma_{D}} \omega \phi \mathrm{d} S
$$

and the weak form of mechanical and electrostatic equilibrium is

$$
0=\delta H=\int_{\Omega}\left(\hat{\sigma}_{i j} \delta \varepsilon_{i j}+\widetilde{\sigma}_{i j k} \delta \varepsilon_{i j, k}-\hat{D}_{i} \delta E_{i}\right) \mathrm{d} \Omega-\int_{\Gamma_{t}} \bar{t}_{i} \delta u_{i} \mathrm{~d} S+\int_{\Gamma_{D}} \omega \delta \phi \mathrm{d} S .
$$

Eq. (15) is the foundation for the computations presented later. The electric potential and mechanical displacement fields, $\phi$ and $\mathbf{u}$, as well as their variations, are approximated with the local maximum-entropy basis functions and the usual Galerkin procedure is carried out

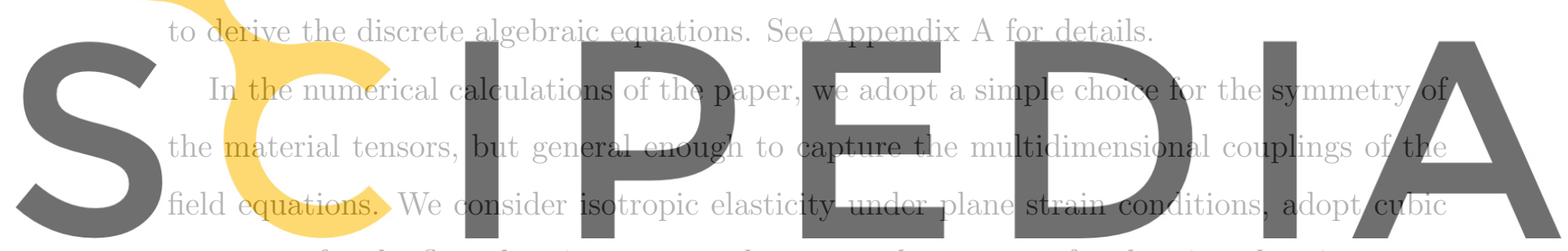

symmetry for the flexoelectric tensor, and tetragonal symmetry for the piezoelectric tensor,

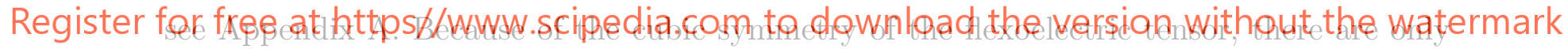

three independent components, $\mu_{1111}, \mu_{1221}$, and $\mu_{1212}$ (or in matrix notation $\mu_{11}, \mu_{12}$, and $\left.\mu_{44}\right)^{21,48}$. In the paper, we only consider the longitudinal and transversal coefficients, $\mu_{11}$ and $\mu_{12}$, since the shear coefficient $\mu_{44}$ is poorly characterized.

\section{NUMERICAL RESULTS}

\section{A. Cantilever beam}

We consider cantilever beams under a point load and different electrical boundary conditions, as depicted in Fig. 1. The aspect ratio of the beam is fixed to $L / h=20$ unless otherwise stated, where $L$ and $h$ are the length and height of the beam. The two electrical boundary conditions considered here are termed open circuit and closed circuit configurations. In the open circuit configuration, we assume that the right-end of the beam is connected to the ground, i.e. the electric potential is fixed to zero, while other sides are 

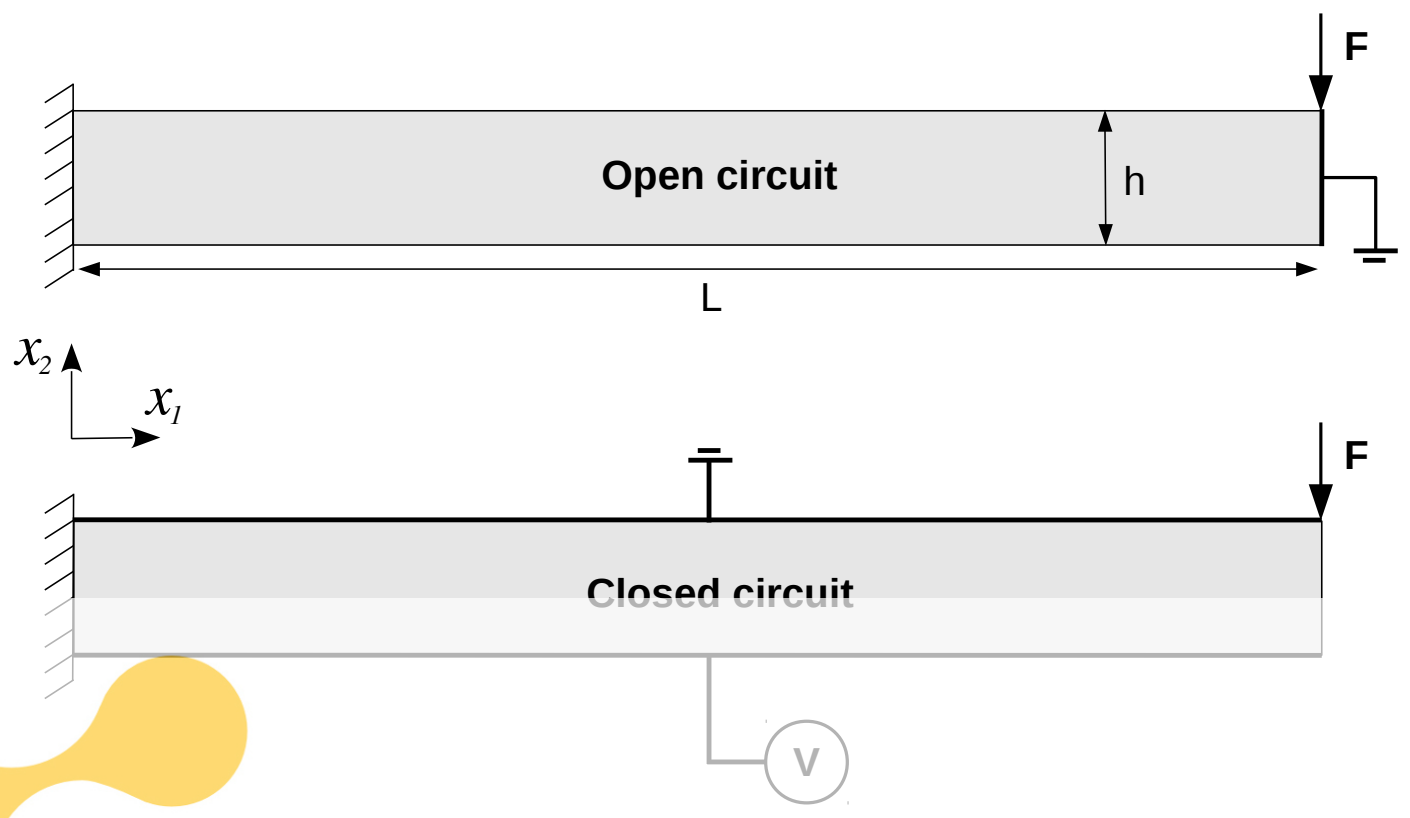

FIG. 1. Electro-mechanical boundary conditions of model cantilever beams. The beams are me-

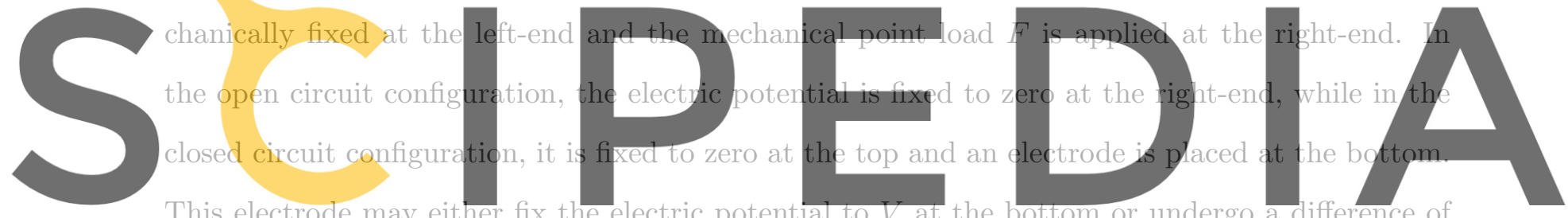

This electrode may either fix the electric potential to $V$ at the bottom or undergo a difference of

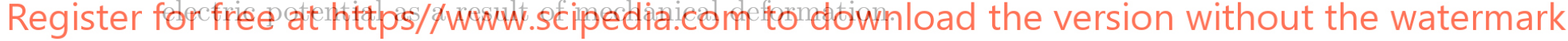

charge free $(\omega=0$ in Eq. (9)). In the closed circuit configuration, two electrodes are attached to the sample at the top and bottom faces. The top electrode is connected to ground, and the bottom electrode can either prescribe a voltage difference $V$, or undergo a change of electric potential as a result of deformation. In this case, we constrain the electric potential to a constant but initially unknown value, which is found as a result of the numerical calculation using Lagrange multipliers. The material parameters are chosen to fit the behavior of single crystals of barium titanate $\left(\mathrm{BaTiO}_{3}\right)$.

\section{Mechanical loading}

The cantilever beams presented in Fig. 1 can convert the mechanical energy induced by the point load into the electrical energy due to electromechanical coupling of the material. 
This energy conversion can be represented by the electromechanical coupling factor $k_{e f f}$

$$
k_{\text {eff }}^{2}=\frac{W_{\text {elec }}}{W_{\text {mech }}}=\frac{\frac{1}{2} \int \mathbf{E} \cdot \kappa \mathbf{E}}{\frac{1}{2} \int \varepsilon: \mathbb{C}: \varepsilon},
$$

where the numerator indicates the total electrical or electrostatic energy and the denominator presents the total elastic energy. Considering both piezoelectricity and flexoelectricity for the open circuit cantilever beam, an analytical estimation for $k_{\text {eff }}$ is presented in Ref. ${ }^{17}$ as

$$
k_{e f f}=\frac{\chi}{1+\chi} \sqrt{\frac{K}{Y}\left(e^{2}+12\left(\frac{\mu}{h}\right)^{2}\right)},
$$

where $\chi=\chi_{33}$ is the electric susceptibility, $K=\kappa_{33}$ is the dielectric constant, $Y$ is the Young's modulus, $e=e_{31}=e_{311}$ is the transversal piezoelectric constant, and $\mu=\mu_{12}$ is the transversal flexoelectric constant. To obtain this formula, it is assumed that the only non-zero components of the stress and electric field are $\sigma_{11}$ and $E_{2}$, respectively. Then, the

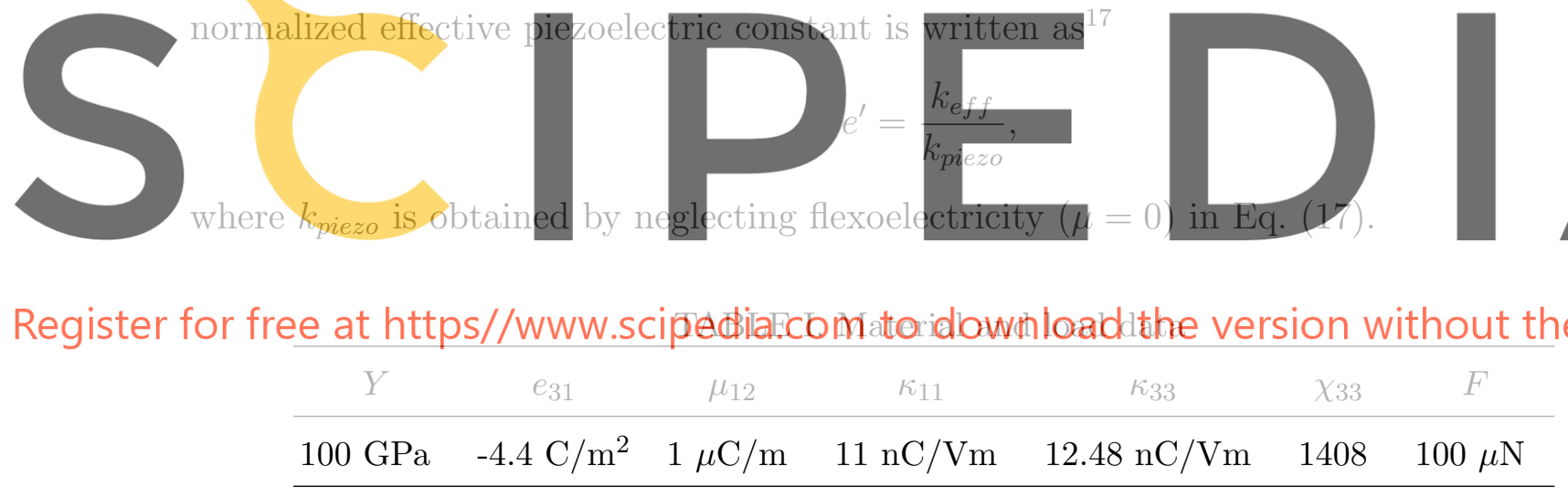

To reproduce computationally the one-dimensional analytical model in Ref. ${ }^{17}$, we consider a simplified model in which only the transversal piezoelectric and flexoelectric constants $e_{31}$ and $\mu_{12}$ are non-zero. Therefore, this model neglects the flexoelectric energy associated with the horizontal component of the electric field $E_{1}$. We also neglect Poisson's effect, i.e. $\nu=0$. Based on the experimental data ${ }^{49}$, the flexoelectric coefficients of $\mathrm{BaTiO}_{3}$ can be estimated to the order of $0.1-1 \mu \mathrm{C} / \mathrm{m}$ in the paraelectric phase, close to the phase transition ${ }^{3}$. For the simulations, we choose $\mu_{12}=1 \mu \mathrm{C} / \mathrm{m}$. With these assumptions and using the material and load data presented in Tab. I, the normalized effective piezoelectric constant $e^{\prime}$ is obtained as a function of the normalized beam thickness $h^{\prime}=-e h / \mu$ for the open circuit condition. Figure 2 presents the results using the analytical formula in Eq. (17) and the present model. It is clear that the electromechanical response of the beam is enhanced by decreasing the 


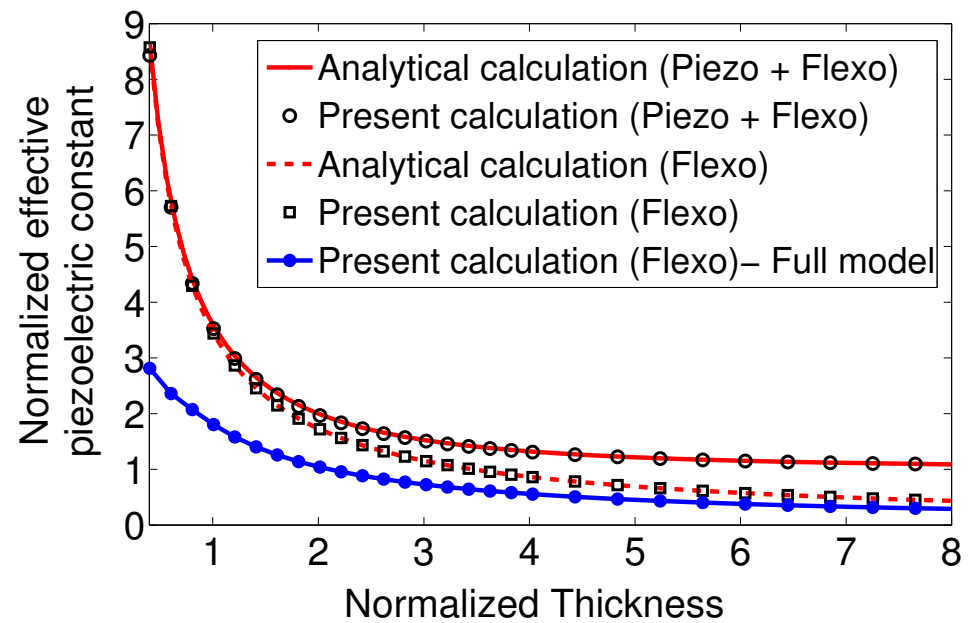

FIG. 2. Normalized effective piezoelectric constant $e^{\prime}$ as a function of the normalized beam thickness $h^{\prime}$ (see text) for piezoelectric and non-piezoelectric materials. The figure compares the analytical formula in Eq. (17) and numerical simulations for the open circuit configuration, both under the simplifying assumptions described in the text and without these assumptions (blue curve).

beam thickness due to flexoelectricity. At larger scales, the effect of flexoelectricity vanishes and the response of the beam converges to that of purely piezoelectric one, i.e. $e^{\prime}=1$. Figure 2 also presents the results for a non-piezoelectric material by setting $e_{31}=0$. Even though the material is not piezoelectric, we can observe a large electromechanical response of the beam at small scales. However, as expected, this response decreases by increasing the beam thickness. An excellent agreement is observed between the analytical and the present calculations, validating our numerical approach.

To assess the effect of the simplifying assumptions behind Eq. (17), we perform simulations with a more general model including the two-dimensional couplings. We consider $\nu=0.37$, and in addition to the transversal flexoelectric coefficient $\mu_{12}$, we also consider the flexoelectric energy terms associated with the longitudinal coefficient $\mu_{11}$, which is chosen to be equal to $\mu_{12}$ in Tab. I. The normalized effective piezoelectric constant of this full model for a non-piezoelectric material is also presented in Fig. 2 as a function of the normalized thickness. It is apparent that the analytical formula, by neglecting two-dimensional effects, significantly overestimates the electromechanical response of the beam described by our more realistic model. For beams of smaller aspect ratio, the discrepancy between the analytical formula and the numerical simulations becomes even larger. However, we have checked 
numerically that the discrepancies between the simplified beam model and the simulations with the more realistic model persist (nearly unchanged) when the aspect ratio is further increased to $L / h=40$. From this point on, we report results for the fully two-dimensional model.

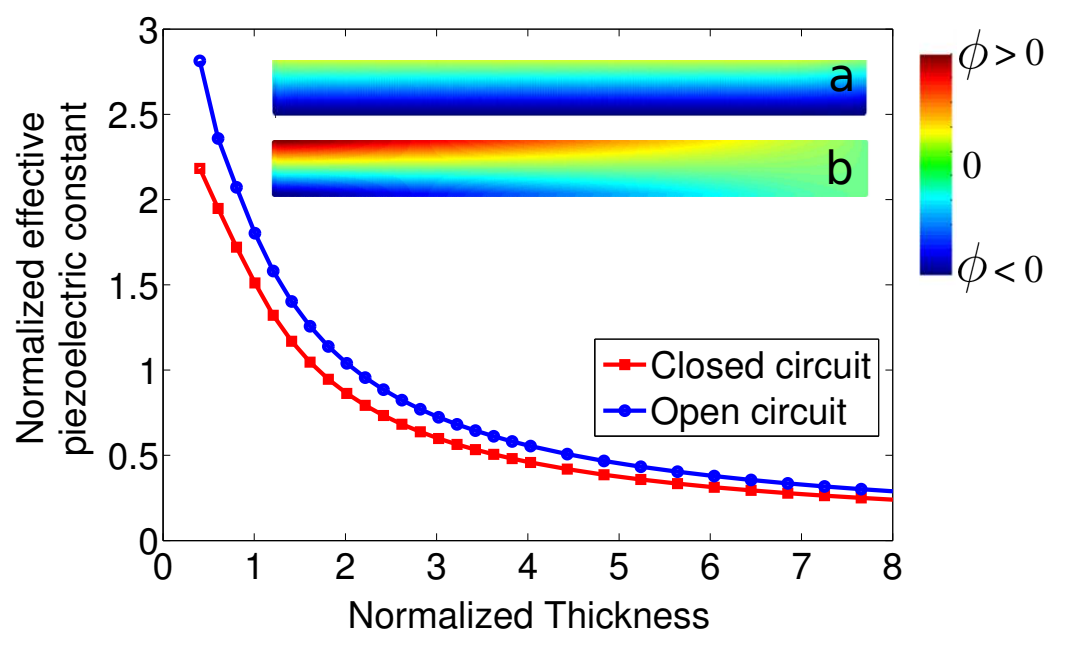

FIG. 3. Normalized effective piezoelectric constant $e^{\prime}$ as a function of the normalized beam thickness $h^{\prime}$ for a non-piezoelectric material in the open and closed circuit configurations, obtained with the present simulation method. The inset shows the distribution of the electric potential $\phi$ in (a) closed and (b) open circuit beams. The aspect ratio has been modified in the plot to better represent the field.

To evaluate the effect of electrical boundary conditions on the electromechanical response of the beam, the results under open and closed circuit configurations are presented in Fig. 3. In the closed-circuit configuration, we set free the potential of the lower electrode. The enhancement of the electromechanical coupling at small scales is stronger for the open circuit configuration. Thus, the open circuit configuration seems to be better for energy conversion. A non-uniform electric potential distribution is observed along the open circuit beam, in which the potential difference or electromechanical response reaches its maximum at the left-end support. This is the point where the beam curvature (the strain gradient) is maximum, thus leading to the maximum flexoelectric effect. Interestingly, a similar non-uniform response has been reported by locally probing the piezoresponse in buckled PZT ribbons ${ }^{50}$. In contrast, the closed circuit beam exhibits a nearly uniform potential difference along the beam as a result of the boundary conditions. 


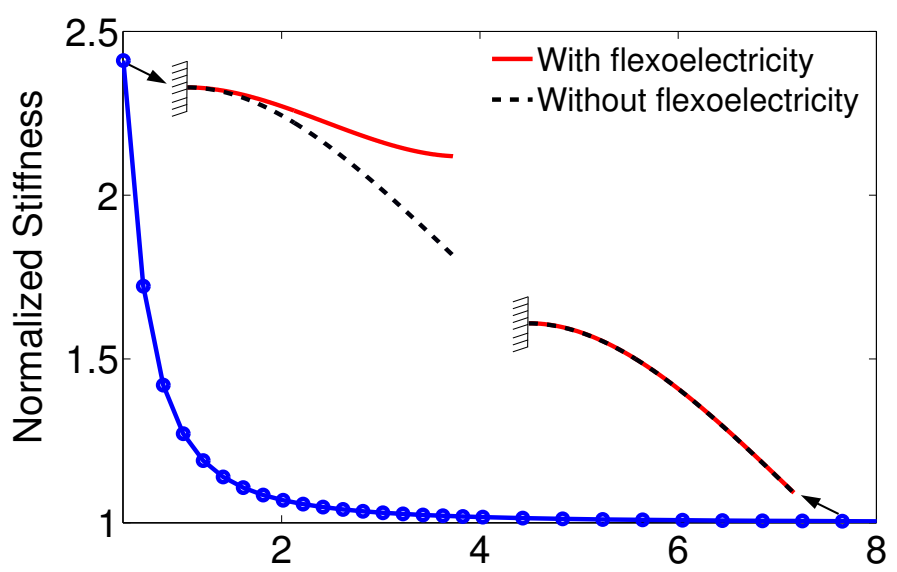

(a)

Normalized Thickness

(i)

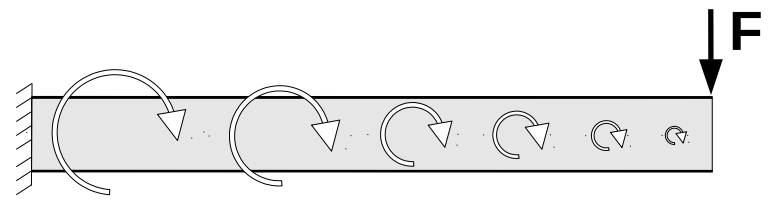

(ii)

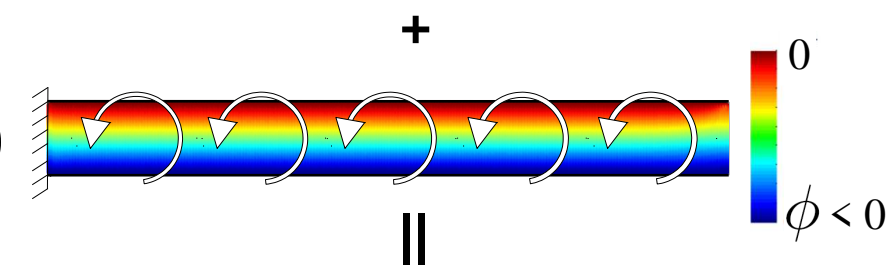

(iii)

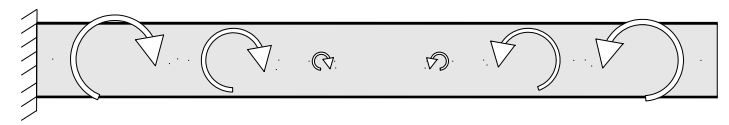

(b)

(iv)
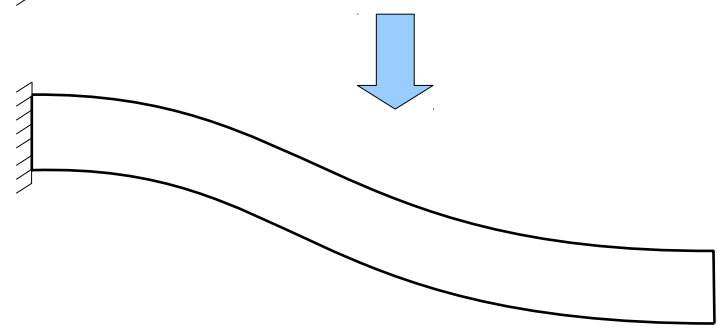

FIG. 4. (a) Normalized stiffness $Y^{\prime}$ as a function of the normalized thickness $h^{\prime}$ for a nonpiezoelectric material considering the closed circuit configuration. The insets show the deformation of the midline of the beam at two different length-scales, with and without flexoelectricity. The same mechanical load is applied for all the simulations in the figure. (b) Illustration of the deformation mechanism of the cantilever flexoelectric beam at small scales. The circular arrows show the moments induced by (i) the mechanical point load $F$ and (ii) the converse flexoelectric effect. Due to the nearly uniform distribution of the electric potential along the beam, a uniform moment is induced due to the converse flexoelectric effect. (iii) The total moment distribution as the summation of the moments in (i) and (ii). The total moments lead to a peculiar deformation of the beam in (iv). The deformation is exaggerated for clarity. 
Another reported manifestation of flexoelectricity is the size-dependent effective elasticity ${ }^{16}$. We evaluate this effect by defining the normalized effective stiffness as:

$$
Y^{\prime}=\frac{\frac{1}{2} \int \varepsilon_{e}: \mathbb{C}: \varepsilon_{e}}{\frac{1}{2} \int \varepsilon_{f}: \mathbb{C}: \varepsilon_{f}},
$$

where $\varepsilon_{f}$ and $\varepsilon_{e}$ are the strains obtained from the simulations of the model with and without considering flexoelectricity, respectively. Figure 4(a) presents $Y^{\prime}$ as a function of the normalized thickness $h^{\prime}$. An enhanced elasticity is apparent when the beam thickness decreases. Flexoelectricity makes the beam stiffer against the applied load at small scales. This effect is vividly shown by plotting the deformation of the beam with and without flexoelectricity (insets). It is apparent that, at small scales, the beam with the effect of flexoelectricity undergoes a smaller deflection than the beam without this effect under the same mechanical load. A similar size effect on the elastic behavior of ferroelectrics due to flexoelectricity has been reported ${ }^{51}$.

The particular deformation of the beam due to flexoelectricity observed in the inset of Fig. 4(a) is examined in Fig. 4(b). We show the moments induced by the mechanical point load $F$ and converse flexoelectricity in (i) and (ii). The moment due to the mechanical load increases linearly from zero at the right-end side of the beam to its maximum value at the left-end side. The mechanical load results in a nearly uniform electric potential distribution along the beam due to direct flexoelectricity, see Fig. 3. The converse flexoelectricity, in turn, induces a uniform distribution of bending moment along the beam, acting against the mechanical load, (ii). Since the flexoelectric moments oppose those mechanically induced, the total moment decreases and even changes sign close to the right-end of the beam (iii). This distribution of moments explains the particular deformation of the beam (iv). This figure, in particular (ii), also suggests studying the actuation induced by flexoelectricity under an applied voltage difference $V$. We explore this point in the following Section.

\section{Electrical loading}

To investigate the electromechanical response of the cantilever beam under purely electrical loading, the mechanical point load $F$ is set to zero and the voltage $V$ is applied to the bottom side of the closed circuit model, see Fig. 1. This model is inspired in the work of Bursian and Zaikovskii ${ }^{49}$, who performed experiments on thin films of single crystal $\mathrm{BaTiO}_{3}$. 
In these experiments, electrodes were deposited on the top and bottom sides of the film to apply a constant through-thickness electric field with a magnitude of $|E|=8 \mathrm{MV} / \mathrm{m}$. To apply this electrical load in the simulations, we set $V=-8 h(\mathrm{MV})$, where $h$ in the beam thickness. Simulation results show that the beam is deflected under the applied electrical load, and thus a cantilever beam can deform as an electromechanical actuator due to flexoelectricity. Figure 5 shows the curvature of the beam as a function of the beam thickness, together with the experimental data in Ref. ${ }^{49}$. A good agreement is obtained between the numerical and experimental results. The curvature $\kappa$ depends strongly on the thickness $h$ and closely follows

$$
\kappa=k h^{-2}
$$

where $k$ is a constant depending on the material properties. From Fig. 5, we obtain $k=0.6$ $\mathrm{nm}$. Note that the sign of the beam curvature is reversed by reversing the direction of the electric field, which was also reported in Ref. ${ }^{49}$. Bending is always in the direction of the positive electrode. Since in the thin beam assumption $\kappa \propto h^{2} M$, we conclude that the bending moment induced by flexoelectricity scales as $h^{-4}$.

This actuation mechanism is analogous to that behind the enhancement of elasticity discussed earlier. To closely examine its emergence, the distribution of the electric field across the thickness of the beam is shown in Fig. 5(b). This distribution is nearly uniform along the entire length of the beam. However, the electric field is very non-uniform across the thickness, and considerable gradients are apparent near the surfaces of the beam (top and bottom sides). These gradients generate high stresses because of converse flexoelectricity, see Eq. (6). Since the signs of the gradients are opposite, the directions of the induced stresses are also opposite, generating a clockwise mechanical moment of actuation. This electrically-induced deformation due to flexoelectricity has been introduced in models for

flexoelectric plates ${ }^{3,14,26}$, which describe in a simplified manner the physics that naturally emerge here by solving the flexoelectric boundary value problem.

\section{B. Truncated pyramid}

Another setup to quantify the flexoelectric response of dielectric solids is the compression of a truncated pyramid. The geometry of the truncated pyramid in plane strain and its boundary conditions are shown in Fig. 6(a). A force of magnitude $F$ is applied uniformly at 


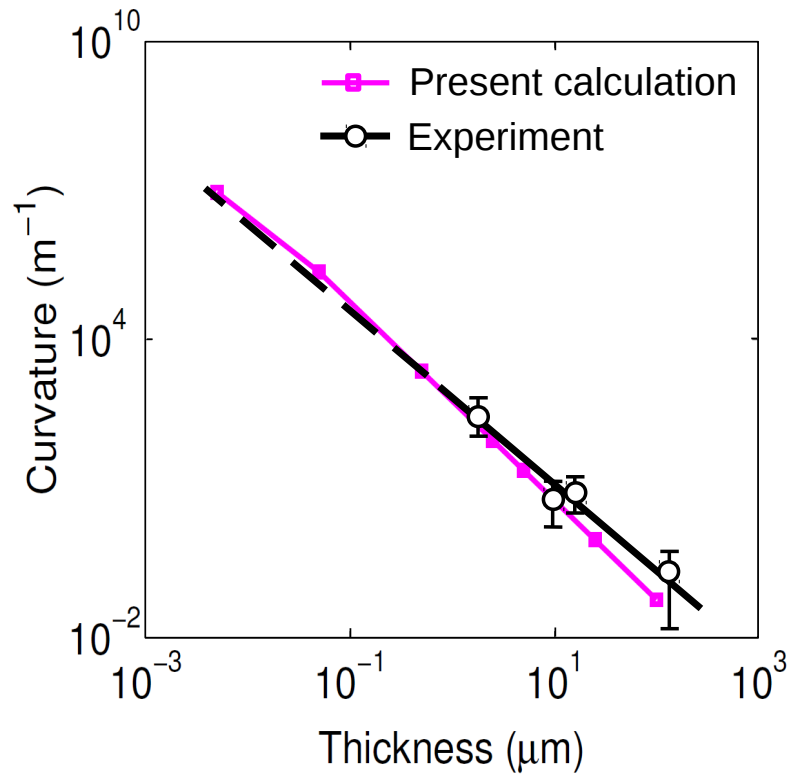

(a)

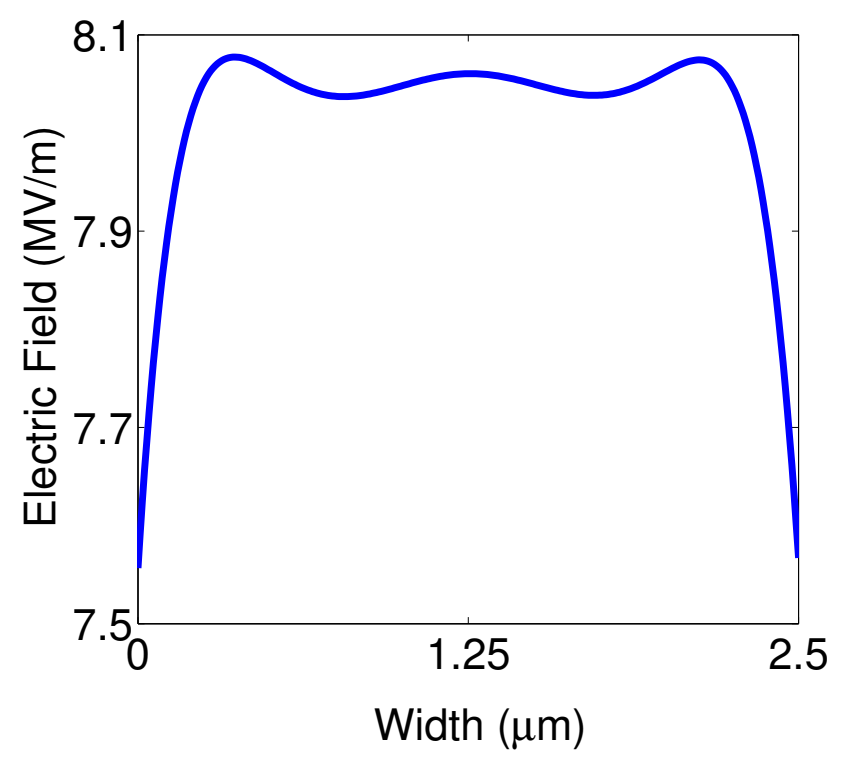

(b)

FIG. 5. (a) Curvature of a cantilever beam as a function of the beam thickness under purely electrical loading. The experimental data are reproduced from the work of Bursian and Zaikovskii ${ }^{49}$. The dashed line indicates the extrapolation of experimental data to hypothetical thinner films. (b) Distribution of the electric field across the beam thickness. Considerable gradients of the electric field near the beam surface are responsible for the beam deflection. 
the top surface. The top and bottom surfaces have areas $a_{1}$ and $a_{2}$. Due to their different areas, the applied force generates different tractions at the top and bottom surfaces, resulting in a longitudinal strain gradient and thus generating a flexoelectric polarization. Here, we focus on a two-dimensional problem by considering a truncated triangle with unit width. If the material has only the longitudinal flexoelectric coefficient $\mu_{11}$ and the elastic constant $c_{11}$, then the longitudinal strain $\varepsilon_{22}$ is the only non-zero strain component, and the effective piezoelectric constant $e_{33}$ of the truncated triangle can be obtained $\mathrm{as}^{21}$ :

$$
e_{33}=\mu_{11}\left(\frac{a_{2}-a_{1}}{h a_{1}}\right)
$$

It is clear that these assumptions are simplistic and in practice, the strain and electric field gradients can be strongly inhomogeneous, particularly near the pyramid corners. To investigate this point, we perform simulations using the model in Fig. 6(a) and the material parameters of $\mathrm{BaTiO}_{3}$ in Tab. I. We consider a non-piezoelectric material with the flexoelectric constants $\mu_{12}=\mu_{11}$. The aspect ratio is chosen as $h=a_{1}=a_{2} / 3$, where $h$ is the height of the truncated triangle. The electric potential is fixed to zero at the top and to a constant but a priori unknown value $V$ at the bottom electrode, as discussed earlier. This model is inspired in the work of Cross and coworkers ${ }^{21,22,52}$, where they performed experiments on an array of truncated pyramids under compression. In these experiments, electrodes were deposited on the top and bottom sides of the pyramids to collect the induced charges. With the resulting $V$, the effective piezoelectric constant $e_{33}$ can be calculated as

$$
e_{33}=\frac{\kappa_{33} E_{2}}{\varepsilon_{22}}=-\frac{\kappa_{33} V a_{2} c_{11}}{h F} .
$$

To derive Eqs. (21) and (22), it is assumed that the flexoelectric truncated triangle mimics the behavior of a piezoelectric rectangle with piezoelectric constant $e_{33}$, elastic constant $c_{11}$, dielectric constant $\kappa_{33}$, width $a_{2}$, height $h$ and under load $F$.

We consider two sets of mechanical boundary conditions at the bottom surface. The first assumption is that the bottom support is fully flexible, i.e. the applied force $F$ induces a uniform traction on the bottom face as in Fig. 6(a). The second assumption considers a rigid support, which prevents the vertical movement of the bottom side, i.e. $u_{2}=0$. In this situation, a non-uniform traction is induced on the bottom surface. Figure 6(b) presents the results for the normalized effective piezoelectric constant $e^{\prime}=e_{33} / e_{0}$ as a function of the normalized height $h^{\prime}=h / h_{0}$, considering the normalization parameters $e_{0}=4.4 \mathrm{Cm}^{-2}$ and 
$h_{0}=75 \mu \mathrm{m}$. These graphs are obtained using Eq. (21) for the estimate of the simplified model and Eq. (22) for the present computations. As in the beam's case, the electromechanical response is enhanced by decreasing the size of the truncated triangle. This size-dependent enhancement is expected from the analytical formula in Eq. (21). However, the results of the present model indicate that the simplified model underestimates the flexoelectric effect. This underestimation is less significant for the rigid support configuration. Similar to the cantilever beam, the difference between the analytical and computational results are due to the two-dimensional effects, which are neglected in the one-dimensional solution in Eq. (21). This point can be clarified further by presenting the distribution of the electric potential and longitudinal strain $\varepsilon_{22}$. These results are obtained for $h=750 \mu \mathrm{m}$ and $F=4.5 \mathrm{KN}$. Figure 6(c) indicates a homogeneous distributions of the gradients of these quantities for the simplified model, while strongly inhomogeneous distributions are observed in Fig. 6(d) and (e) for the present model with the flexible and rigid support conditions. In addition, we observe that the deformation mode of the pyramid with the flexible support includes a bending component. It is clear in Fig. 6(d) that due to the bending, the strain changes sign with respect to Fig. 6(c). This is not the case for Fig. 6(e) since the rigid support avoids the bending of the pyramid. Therefore, the origin of the difference between the analytical and computational results is mainly the bending deformation mode of the pyramid. Another source of this difference is sharp changes of the strain and electric potential near the sample corners, resulting in a flexoelectric effect. These results suggest that simple estimations such as those leading to Eq. (21) are not reliable to compute the flexoelectric response of truncated pyramids. Since Eq. (21), used to quantify the flexoelectric constants of dielectric solids from experimental data ${ }^{21}$, underestimates the flexoelectric effect, the reported flexoelectric constants are overestimated, which may at least partially explain the discrepancies between experimental measurements and theoretical estimates ${ }^{3}$. Our results also highlight the importance of boundary conditions on the flexoelectric response. Computationally, the truncated pyramid problem is more challenging that the cantilever beam, since the complex fields near the corners need to be resolved to accurately evaluate the effective piezoelectricity of the system, see Appendix B for a convergence study.

Finally, we note that the minimal normalized thicknesses we have considered for the cantilever beam and the truncated pyramid are $h^{\prime}=0.4$ and $h^{\prime}=0.05$, respectively. Below these scales, the model predicts that the effective piezoelectric constant decreases with size, 
which is not expected from flexoelectricity. This counterintuitive behavior at such small scales is a result of the non-positive definiteness of the energy. As suggested in Ref. ${ }^{35}$, including strain-gradient elasticity in the model is crucial for the positive-definiteness of the energy. However, discussing beam bending, it is argued that with the material parameters of barium titanate $\left(\mu_{12}=63 \mathrm{nC} / \mathrm{m}\right)$, the bending rigidity would only become negative for beam thicknesses below $5.39 \mathrm{~nm}^{23}$. According to our calculations with the same material parameters, we obtain a critical thickness for loss of stability of $5.7 \mathrm{~nm}$. At these small scales, the continuum model that we are following is insufficient. Leaving aside that atomistic simulations may be more adequate in some cases, a continuum model at these scales should include strain gradient elasticity and surface effects, such as surface piezoelectricity ${ }^{14}$.

\section{CONCLUSIONS}

We have addressed computationally the flexoelectric response in dielectric solids, resorting to smooth local maximum-entropy (LME) meshfree approximants to deal with the highorder coupled PDE. By considering two standard test examples, bending of a cantilever beam and compression of a truncated pyramid, we have tested our numerical approach against simplified solutions of the flexoelectric boundary value problem, and highlighted the quantitative limitations of such approximations. We have shown that the proper treatment

of the multi-dimensionality of the problem is important to accurately evaluate the effect of flexoelectricity, which is otherwise under- or over-estimated by simplified models. We have also shown the critical role of boundary conditions.

Our simulations reproduce well-known features of flexoelectricity, such as the sizedependent enhancement of piezoelectricity. The simulations provide insights into the enhanced elasticity of cantilever flexoelectric beams and their deformation modes as size shrinks. We have also analyzed the flexoelectric actuation of beams by electric fields, obtaining a scaling of the beam curvature with size previously reported in experiments. We have found that the source of actuation is a high gradient of electric field near the beam surface, which induces a moment due to the converse flexoelectric effect. Our simulations on the truncated pyramid system further highlight the need for multi-dimensional treatments that accurately account for boundary conditions to properly understand and evaluate flexoelectricity. This example shows the strong effects due to a bending component of the 
(a)

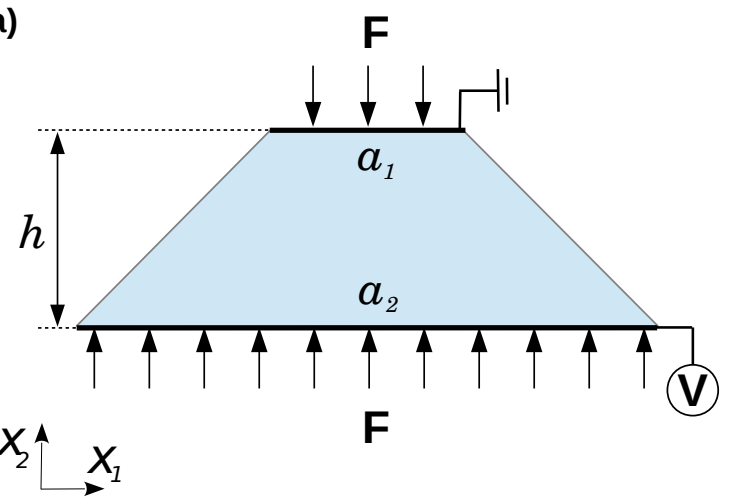

(b)

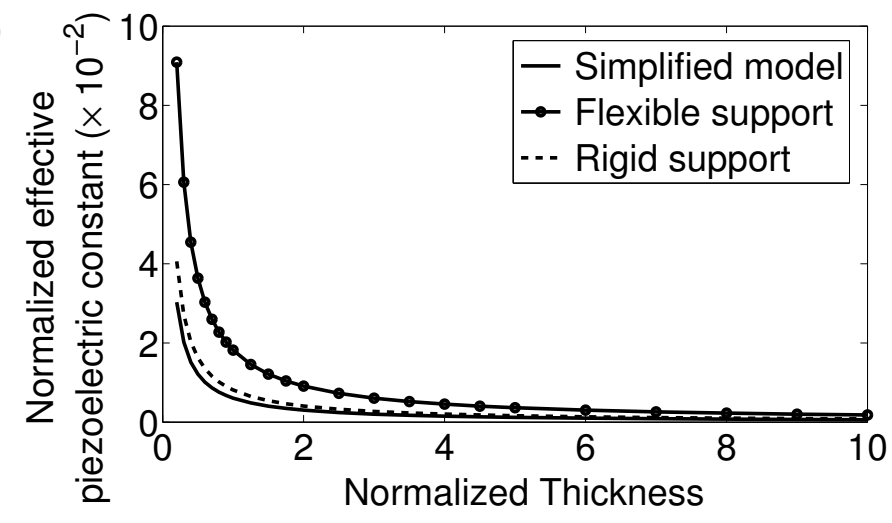

(c) Simplified model

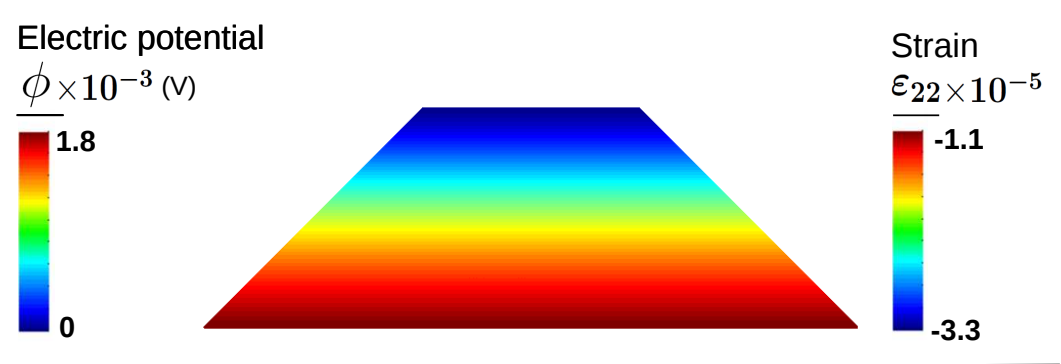

(d) Flexible support

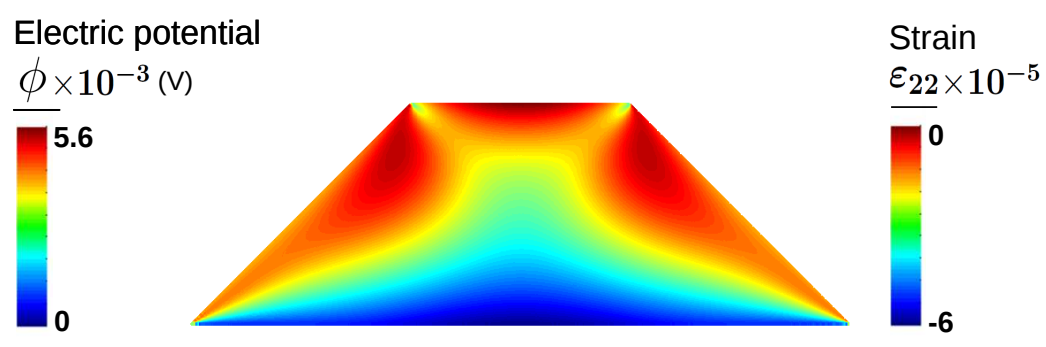

(e) Rigid support

Electric potential

Strain

$\phi \times 10^{-3}(\mathrm{~V})$
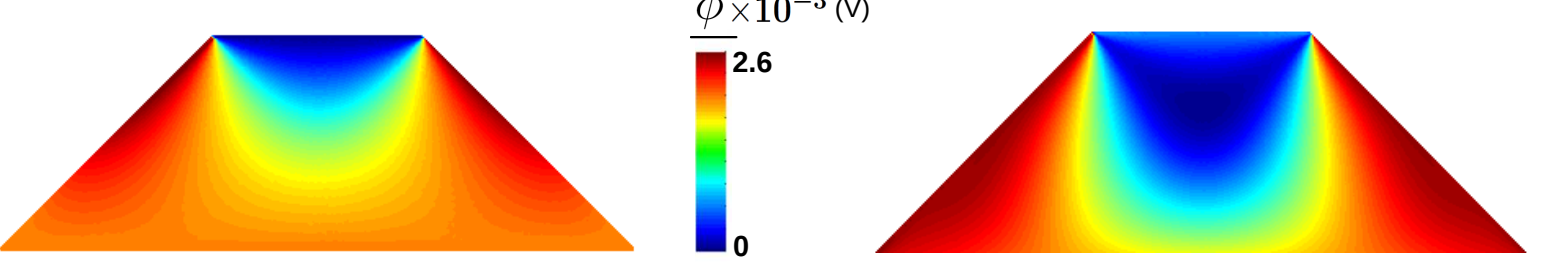

$\varepsilon_{22} \times 10^{-5}$

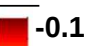

41

FIG. 6. (a) Truncated pyramid in plane strain under the mechanical load $F$, uniformly distributed at the top surface. The top face has length $a_{1}$ and the bottom length $a_{2}$. The electric potential is fixed to zero at the top and is constant but unknown at the bottom. (b) Normalized effective piezoelectric constant $e^{\prime}$ as a function of the normalized thickness $h^{\prime}$ for a non-piezoelectric material, (c-e)(left) Distribution of the electric potential and (right) the strain $\varepsilon_{22}$ obtained from the simplified analytical model (c) and the computational models with the flexible (d) and rigid (e) supports. 
pyramid deformation and geometric features of the domain, such as corners. The resulting flexoelectric response indicates that a simple analytical formulae commonly used to interpret truncated pyramid experiments underestimates the flexoelectric effect.

Our results suggest that actuators or energy-harvesting devices based on flexoelectricity can be optimized to achieve significantly better performance by properly designing their geometry and boundary conditions, including the mechanical confinement and electrode configuration. For this purpose, computational techniques are very valuable to guide experimental implementations.

\section{ACKNOWLEDGMENT}

The authors gratefully acknowledge the support of the Ministerio de Ciencia e Innovación (DPI2011-26589).

\section{APPENDIX A: NUMERICAL APPROXIMATION}

In recent years, a new sort of polygonal approximants ${ }^{53}$ and meshfree approximation schemes ${ }^{42}$ have been developed based on the information theoretic concept of maximumentropy. Essentially, these methods allows us to determine a set of smooth basis functions $p^{a}(\mathbf{x})$, each localized around its corresponding node of the grid. In particular, we follow the LME approximants as detailed in Ref. ${ }^{54}$, and expand the continuum fields as

$$
\mathbf{u}(\mathbf{x})=\sum_{a=1}^{N} p^{a}(\mathbf{x}) \mathbf{u}^{a}, \quad \phi(\mathbf{x})=\sum_{a=1}^{N} p^{a}(\mathbf{x}) \phi^{a} .
$$

From now on, we ignore the arguments of the basis functions and nodal values for simplicity, i.e. $\mathbf{u}=\sum_{a=1}^{N} p^{a} \mathbf{u}^{a}$. We thus have

$$
\partial_{j} u_{i}=\sum_{a=1}^{N} \partial_{j} p^{a} u_{i}^{a}, \quad \partial_{j} \partial_{k} u_{i}=\sum_{a=1}^{N} \partial_{j} \partial_{k} p^{a} u_{i}^{a}, \quad \partial_{j} \phi=\sum_{a=1}^{N} \partial_{j} p^{a} \phi^{a} .
$$

Note that these terms involve the gradient and Hessian of the LME basis functions.

Plugging the discrete representation into the total electromechanical enthalpy in Eq. (14), we obtain the algebraic function in terms of the nodal displacements and electric potential 
degrees of freedom

$$
\begin{aligned}
H(\mathbf{U}, \phi)= & \frac{1}{2} \sum_{a, b} \mathbf{u}^{a T}\left(\int_{\Omega} \mathbf{B}_{\mathbf{u}}\left(p^{a}\right) \mathbb{C B}_{\mathbf{u}}^{T}\left(p^{b}\right) d \Omega\right) \mathbf{u}^{a} \\
& +\sum_{a, b} \mathbf{u}^{a T}\left(\int_{\Omega} \mathbf{B}_{\mathbf{u}}\left(p^{a}\right) \mathbf{e} \mathbf{B}_{\phi}^{T}\left(p^{b}\right) d \Omega\right) \phi^{b} \\
& +\sum_{a, b} \mathbf{u}^{a T}\left(\int_{\Omega} \mathbf{H}_{\mathbf{u}}\left(p^{a}\right) \mu^{T} \mathbf{B}_{\phi}^{T}\left(p^{b}\right) d \Omega\right) \phi^{b} \\
& -\frac{1}{2} \sum_{a, b}\left(\int_{\Omega} \mathbf{B}_{\phi}\left(p^{a}\right) \mathbf{K B}_{\phi}^{T}\left(p^{b}\right) d \Omega\right) \phi^{a} \phi^{b} \\
& -\sum_{a}\left(\int_{\Gamma_{t}} \overline{\mathbf{t}} p^{a} \mathrm{~d} S\right) \mathbf{u}^{a}+\sum_{a}\left(\int_{\Gamma_{D}} \omega p^{a} \mathrm{~d} S\right) \phi^{a},
\end{aligned}
$$

where the stiffness tensor $\mathbb{C}$, the dielectric tensor $\mathbf{K}$, the piezoelectric tensor $\mathbf{e}$, and the flexoelectric tensor $\mu$ have been written in Voigt form as

$$
\begin{aligned}
& \mathbb{C}=\frac{Y}{(1+\nu)(1-2 \nu)}\left[\begin{array}{ccc}
1-\nu & \nu & 0 \\
\nu & 1-\nu & 0 \\
0 & 0 & \frac{1-2 \nu}{2}
\end{array}\right] \text {, } \\
& \mathbf{K}=\left[\begin{array}{cc}
\kappa_{11} & 0 \\
0 & \kappa_{33}
\end{array}\right], \quad \mathbf{e}^{T}=\left[\begin{array}{ccc}
0 & 0 & e_{15} \\
e_{31} & e_{33} & 0
\end{array}\right] \text {, } \\
& \mu=\left[\begin{array}{cccccc}
\mu_{11} & \mu_{12} & 0 & 0 & 0 & \mu_{44} \\
0 & 0 & \mu_{44} & \mu_{12} & \mu_{11} & 0
\end{array}\right] \text {. }
\end{aligned}
$$

The gradient operators $\mathbf{B}_{\mathbf{u}}$ and $\mathbf{B}_{\phi}$ and the Hessian operator $\mathbf{H}_{\mathbf{u}}$ can be written in Voigt form as

$$
\begin{aligned}
& \mathbf{B}_{\mathbf{u}}=\left[\begin{array}{ccc}
\frac{\partial}{\partial x} & 0 & \frac{\partial}{\partial y} \\
0 & \frac{\partial}{\partial y} & \frac{\partial}{\partial x}
\end{array}\right], \quad \mathbf{B}_{\phi}=\left[\begin{array}{cc}
\frac{\partial}{\partial x} & \frac{\partial}{\partial y}
\end{array}\right], \\
& \mathbf{H}_{\mathbf{u}}=\left[\begin{array}{cccccc}
\frac{\partial^{2}}{\partial x^{2}} & 0 & \frac{\partial^{2}}{\partial y \partial x} & \frac{\partial^{2}}{\partial x \partial y} & 0 & \frac{\partial^{2}}{\partial y^{2}} \\
0 & \frac{\partial^{2}}{\partial y \partial x} & \frac{\partial^{2}}{\partial x^{2}} & 0 & \frac{\partial^{2}}{\partial y^{2}} & \frac{\partial^{2}}{\partial x \partial y}
\end{array}\right] .
\end{aligned}
$$

Following the usual Galerkin procedure, it is possible to derive the discrete algebraic equations for the equilibrium as

$$
\left[\begin{array}{cc}
\mathbf{A}_{\mathbf{U U}} & \mathbf{A}_{\mathbf{U} \phi} \\
\mathbf{A}_{\phi \mathbf{U}} & \mathbf{A}_{\phi \phi}
\end{array}\right]\left[\begin{array}{l}
\mathbf{U} \\
\phi
\end{array}\right]=\left[\begin{array}{l}
\mathbf{f}_{\mathbf{U}} \\
\mathbf{f}_{\phi}
\end{array}\right]
$$


where the local contribution of each quadrature point to the matrix of system has the structure

$$
\begin{gathered}
\mathbf{A}_{\mathbf{U U}}^{a b}=\mathbf{B}_{\mathbf{u}}\left(p^{a}\right) \mathbb{C B}_{\mathbf{u}}^{T}\left(p^{b}\right), \\
\mathbf{A}_{\mathbf{U} \phi}^{a b}=\mathbf{B}_{\mathbf{u}}\left(p^{a}\right) \mathbf{e} \mathbf{B}_{\phi}^{T}\left(p^{b}\right)+\mathbf{H}_{\mathbf{u}}\left(p^{a}\right) \mu^{T} \mathbf{B}_{\phi}^{T}\left(p^{b}\right), \\
\mathbf{A}_{\phi \mathbf{U}}^{a b}=\mathbf{B}_{\phi}\left(p^{b}\right) \mathbf{e}^{T} \mathbf{B}_{\mathbf{u}}^{T}\left(p^{a}\right)+\mathbf{B}_{\phi}\left(p^{b}\right) \mu \mathbf{H}_{\mathbf{u}}^{T}\left(p^{a}\right), \\
\mathbf{A}_{\phi \phi}^{a b}=-\mathbf{B}_{\phi}\left(p^{a}\right) \mathbf{K} \mathbf{B}_{\phi}^{T}\left(p^{b}\right), \\
\mathbf{f}_{\mathbf{U}}=\overline{\mathbf{t}} p^{a}, \mathbf{f}_{\phi}=-\omega p^{a},
\end{gathered}
$$

where the basis functions derivatives are evaluated at the corresponding each quadrature point.

\section{APPENDIX B: CONVERGENCE ANALYSIS}

As in other numerical techniques to approximate boundary value problems, we perform here a convergence analysis to assure the accuracy of the results. We perform a number of simulations for both the cantilever beam and the truncated pyramid with fixed normalized sizes of $h^{\prime}=4$ and $h^{\prime}=10$, respectively. For each example, five node sets of variable resolution are considered. The nodes are uniformly distributed for the cantilever beam. To capture the sharp changes of the strain and electric field near the edges of the pyramid, the nodal spacing is chosen to be smaller near the edges than in the bulk. To increase the resolution of each node set, the nodal spacing is decreased by half by inserting an extra node between each pair of closest nodes. Figure 7 presents the normalized effective piezoelectric constant as a function of degrees of freedom (DOF), which is proportional to the number of nodes. Each simulation is performed with different values of $\gamma$, a dimensionless parameter characterizing the degree of locality of the local maximum-entropy basis function. As $\gamma$ increases, the basis functions become sharper and more local. It is obvious from this figure that the results converge to a single solution, irrespective of the choice of $\gamma$ in the range [0.8-1.8]. The convergence is slower for the pyramid since a finer node distribution is required as compared 
to the beam to properly capture the localized effects at the pyramid corners. Note that the computational cost of each simulation increases considerably by decreasing $\gamma$. Based on this convergence analysis and considering a reasonable computational cost, we choose $\gamma=1$ and the node sets of $2.8 \times 10^{4}(\mathrm{DOF})$ and $1.4 \times 10^{5}(\mathrm{DOF})$ for the simulations of Sections III A and III B, respectively. In all simulations, we build a Delaunay triangulation of each node set and generate a standard Gauss-Legendre quadrature rule of 12 points (order 6) per triangle, an overkill integration rule. A tolerance of $T O L_{0}=10^{-8}$ for the cutoff of the neighbor search and a tolerance of $T O L_{N R}=10^{-14}$ for the Newton-Raphson solution of the dual optimization problem are chosen to provide good accuracy at a reasonable computational $\operatorname{cost}^{45}$.
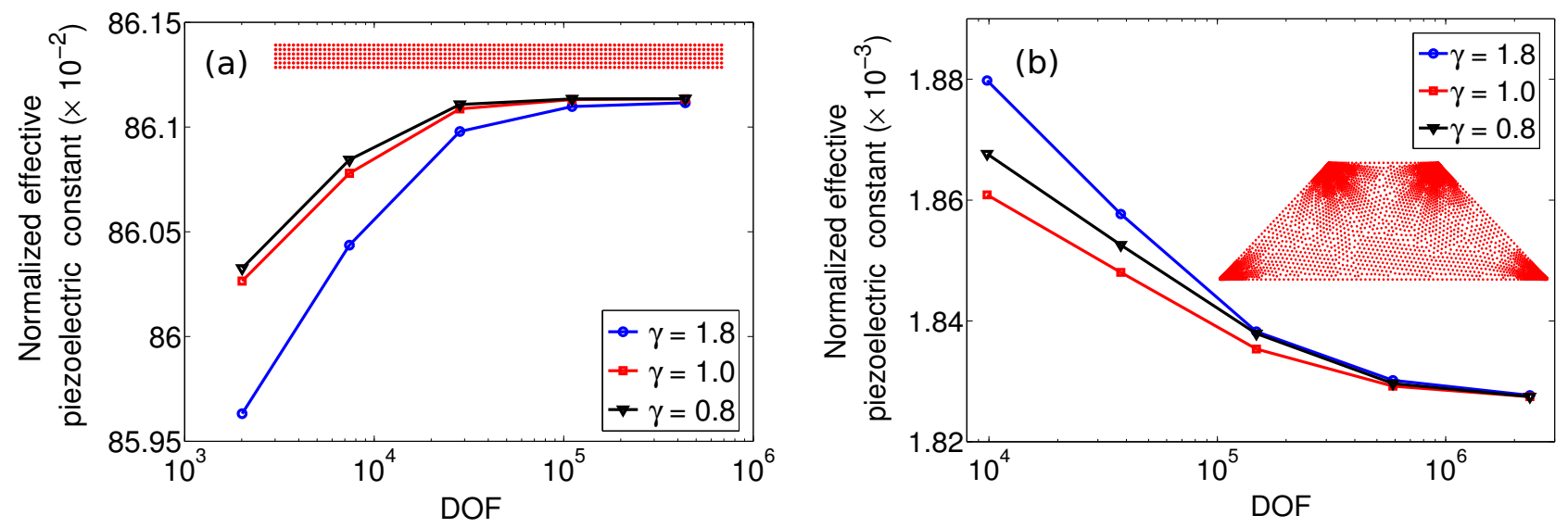

FIG. 7. Normalized effective piezoelectric constant as a function of degrees of freedom (DOF) for (a) the open circuit cantilever beam with a normalized size of $h^{\prime}=4$ and (b) the truncated triangle with a normalized size of $h^{\prime}=10$. In each case, DOF is proportional to the number of nodes and the simulations are performed with five node sets of variable resolution. Each simulation is performed with different values of $\gamma$, a dimensionless parameter characterizing the degree of locality of the LME basis function. The inset shows a representative computational node set for each case.

\section{REFERENCES}

${ }^{1}$ V. S. Mashkevich and K. B. Tolpygo, I. Sov. Phys. JETP 5, 435 (1957).

${ }^{2}$ T. D. Nguyen, S. Mao, Y.-W. Yeh, P. K. Purohit, and M. C. McAlpine, Adv. Mater. 25, $946(2013)$. 
${ }^{3}$ P. Zubko, G. Catalan, and A. K. Tagantsev, Annu. Rev. Mater. Res. 43, 387 (2013).

${ }^{4}$ P. V. Yudin and A. K. Tagantsev, Nanotech. 24, 432001 (2013).

${ }^{5}$ W. Ma and L. E. Cross, Appl. Phys. Lett. 81, 3440 (2002).

${ }^{6}$ G. Catalan, L. J. Sinnamon, and J. M. Gregg, J. Phys.: Cond. Matter 16, 2253 (2004).

${ }^{7}$ W. Ma and L. E. Cross, Appl. Phys. Lett. 86, 072905 (2005).

${ }^{8}$ P. Zubko, G. Catalan, A. Buckley, P. R. L. Welche, and J. F. Scott, Phys. Rev. Lett. 99, 167601 (2007).

${ }^{9}$ W. Ma and L. E. Cross, Appl. Phys. Lett. 88, 232902 (2006).

${ }^{10}$ H. Lu, C.-W. Bark, D. Esque De Los Ojos, J. Alcala, C. Eom, G. Catalan, and A. Gruverman, Science 335, 59 (2012).

${ }^{11}$ S. M. Kogan, Sov. Phys. Solid State 5, 2069 (1964).

${ }^{12}$ A. K. Tagantsev, Phys. Rev. B 34, 5883 (1986).

${ }^{13}$ A. K. Tagantsev, V. Meunier, and P. Sharma, MRS Bulletin 34, 643 (2009).

${ }^{14}$ A. Tagantsev and A. Yurkov, J. Appl. Phys. 112, 044103 (2012).

${ }^{15}$ R. Maranganti, N. D. Sharma, and P. Sharma, Phys. Rev. B 74, 014110 (2006).

${ }^{16}$ M. S. Majdoub, P. Sharma, and T. Cagin, Phys. Rev. B 77 (2008).

${ }^{17}$ M. S. Majdoub, P. Sharma, and T. Cagin, Phys. Rev. B 79 (2009).

${ }^{18}$ N. D. Sharma, R. Maranganti, and P. Sharma, J. Mech. Phys. Solids 55, 2328 (2007).

${ }^{19}$ N. D. Sharma, C. M. Landis, and P. Sharma, J. Appl. Phys. 108, 024304 (2010).

${ }^{20}$ M. S. Majdoub, P. Sharma, and T. Cagin, Phys. Rev. B 78, 121407 (2008).

${ }^{21}$ L. E. Cross, J. Mater. Sci., J. Mater. Sci. 41, 53 (2006).

${ }^{22}$ W. Zhu, J. Y. Fu, N. Li, and L. Cross, Appl. Phys. Lett. 89, 192904 (2006).

23Z. Yan and L. Y. Jiang, J. Appl. Phys. 113, 194102 (2013).

${ }^{24}$ Z. Yan and L. Jiang, J. Phys. D: Appl. Phys. 46, 355502 (2013).

${ }^{25}$ M. C. Ray, J. Appl. Mech. 81, 091002 (2014).

${ }^{26}$ A. E. Eliseev, A. N. Morozovska, M. D. Glinchuk, and R. Blinc, Phys. Rev. B 79, 165433 (2009).

${ }^{27}$ S. Shen and S. Hu, J. Mech. Phys. Solids 58, 665 (2010), ISSN 0022-5096.

${ }^{28}$ A. Askar, P. C. Y. Lee, and A. S. Cakmak, Phys. Rev. B 1, 3525 (1970).

${ }^{29}$ S. V. Kalinin and V. Meunier, Phys. Rev. B 77, 033403 (2008).

${ }^{30}$ R. Maranganti and P. Sharma, Phys. Rev. B 80, 054109 (2009).

${ }^{31}$ T. Xu, J. Wang, T. Shimada, and T. Kitamura, J. Phys. Cond. Matter 25, 415901 (2013). 
${ }^{32}$ J. Hong and D. Vanderbilt, Phys. Rev. B 88, 174107 (2013).

${ }^{33}$ M. Stengel, Phys. Rev. B 88, 174106 (2013).

${ }^{34}$ M. Stengel, Nature Commun. 4, 2693 (2013).

${ }^{35}$ S. Mao and P. K. Purohit, J. Appl. Mech. 81, 081004 (2014).

${ }^{36}$ R. Ahluwalia, A. K. Tagantsev, P. Yudin, N. Setter, N. Ng, and D. J. Srolovitz, Phys. Rev. B 89, 174105 (2014).

${ }^{37}$ Y. Gu, M. Li, A. N. Morozovska, Y. Wang, E. A. Eliseev, V. Gopala, and L.-Q. Chen, Phys. Rev. B 89, 174111 (2014).

${ }^{38}$ H. Chen, A. Soh, and Y. Ni, Acta Mech. 225, 1323 (2014).

${ }^{39}$ J. Shu, W. King, and N. Fleck, Int. J. Numer. Meth. Eng. 44, 373 (1999).

${ }^{40}$ E. Amanatidou and N. Aravas, Comput. Methods. Appl. Mech. Eng. 191, 1723 (2002).

${ }^{41}$ S. Markolefas, D. Tsouvalas, and G. Tsamasphyros, Int. J. Solids Struct. 45, 3255 (2008).

${ }^{42}$ M. Arroyo and M. Ortiz, Int. J. Numer. Meth. Eng. 65, 2167 (2006).

${ }^{43}$ A. Rosolen, C. Peco, and M. Arroyo, J. Comput. Phys. 246, 303 (2013).

${ }^{44}$ C. Peco, A. Rosolen, and M. Arroyo, J. Comput. Phys. 249, 320 (2013).

${ }^{45}$ D. Millán, A. Rosolen, and M. Arroyo, Int. J. Numer. Meth. Eng. 85, 723 (2011).

${ }^{46}$ D. Millán, A. Rosolen, and M. Arroyo, Int. J. Numer. Meth. Eng. 93, 685 (2013).

${ }^{47}$ H. Le Quang and Q. C. He, Proc. Royal Soc. A 467, 2369 (2011).

${ }^{48}$ L. Shu, X. Wei, T. Pang, X. Yao, and C. Wang, J. Appl. Phys. 110, 104106 (2011).

${ }^{49}$ E. V. Bursian and O. I. Zaikovskii, Sov. Phys. Solid State 10, 1121 (1968).

${ }^{50}$ Y. Qi, J. Kim, T. D. Nguyen, B. Lisko, P. K. Purohit, and M. C. McAlpine, Nano Lett. 11, 1331 (2011).

${ }^{51}$ M. Gharbi, Z. H. Sun, P. Sharma, and K. White, Appl. Phys. Lett. 95, 142901 (2009).

${ }^{52}$ J. Y. Fu, W. Zhu, N. Li, N. B. Smith, and L. E. Cross, Appl. Phys. Lett. 91, 182910 (2007).

${ }^{53}$ N. Sukumar, Int. J. Numer. Meth. Eng. 61, 2159 (2004).

${ }^{54}$ A. Rosolen, D. Millán, and M. Arroyo, Int. J. Numer. Meth. Eng. 82, 868 (2010). 